\title{
Análisis de dos Clavos y un rodamiento del pecio Bou Ferrer (LA Vila-Joiosa, Alicante)
}

Anaylisis of two nails and a bearing of the shipwreck Bou Ferrer (La Vila-Joiosa, Alicante)

\section{JOSÉ ANTONIO MOYA}

INAPH - Universitat d'Alacant. jamoya@uas.es (https://orcid.org/0000-0002-9095-8129)

\section{RESUMEN:}

El pecio Bou Ferrer contiene los restos sumergidos de una nave de comercio romana altoimperial que conserva una parte importante de su arquitectura naval. Analizamos tres objetos de metal procedentes del yacimiento, dos clavos y un hipotético rodamiento de la bomba de achique, para conocer la composición elemental.

El resultado ha revelado que los clavos son de cobre y el cojinete de una aleación ternaria de bronce que contiene cobre, estaño y plomo. Este hallazgo abre la posibilidad de investigar el uso especializado del bronce plomado para mejorar el rendimiento mecánico de las bombas de achique en los barcos romanos.

Palabras clave: arqueología subacuática, bombas de achique, metal arqueológico, bronce antiguo.

Abstract:

The Bou Ferrer wreck contains the underwater remains of a high-empire Roman trade ship. This archaeological site preserves an important part of the naval architecture, so we analyse three metal objects in order to know their elementary composition: two nails and a hypothetical bearing from the bilge pump. The results reveal that both nails are made of copper, clearing up the question about their physical appearance. The bearing is made of bronze, but in a ternary alloy containing lead. The chemical composition is similar to equivalent artifacts from the Ben-Afeli archaeological site. This finding opens up the research of a possible specialized use of leaded bronze to improve the mechanical performance of the bilge pumps on ancient roman ships.

Key words: underwater archaeology, bilge pumps, archaeological metal, ancient bronze. 
La investigación en el yacimiento Bou Ferrer, un pecio romano localizado en la costa de Villajoyosa (Alicante), se viene realizando desde su descubrimiento, en el año 2000, mediante diferentes intervenciones arqueológicas anuales promovidas por la Generalitat Valenciana y la colaboración del Ayuntamiento de Villajoyosa. Los primeros trabajos consistieron en un sondeo en la zona central del navío, cubierto por un túmulo de ánforas. Posteriormente se amplió hasta convertirlo en una trinchera de $6 \times 20$ m y 3,5 de profundidad, perpendicular a la quilla, que pasa por la carlinga, y rebasa los restos conservados de los costados de babor y estribor (De Juan et al. 2017). La trinchera ha permitido acceder al casco del barco y ha proporcionado información sobre las técnicas constructivas, la carga de ánforas del tipo Dr. 7-11, y un conjunto de lingotes de plomo. La cronología se ha precisado entre los años 66-68 d.C. gracias a la epigrafía de los lingotes y al hallazgo de un sestercio y un dupondio, ambos del emperador Nerón. También han sido exhumados los tres artefactos metálicos que estudiamos en el presente trabajo, dos clavos cuya apariencia sugería que pudiesen ser de bronce o de cobre, similares a los que describen Galili et al. (2010) en los pecios de Ashkelon y Carmel en la costa de Israel, o Fernández (1980) en el pecio de Ben-Afeli; y una pieza metálica identificada como un rodamiento o cojinete que probablemente formó parte de la bomba de achique, cuyos paralelos conocidos, formalmente similares, son de bronce, por ejemplo Ramos et al. (1984), Beltrame y Gaddi (2005), Carre (2007) y Long (2012). Las fotografías que se muestran fueron realizadas tras la recuperación de las piezas que actualmente se encuentran en proceso de restauración en Vilamuseu.

De los tres artefactos se extrajeron muestras para el análisis con el fin de determinar su composición elemental. El instrumento utilizado es un microscopio electrónico de barrido Hitachi S3000N con detector de RX Bruker XFlash 3001.

Los análisis de los dos clavos (fig. 1) dieron como resultado que el elemento principal en la composición de ambos es el cobre, con valores respectivos de $85.43 \%$ y $88.45 \%$, según se recoge en la tabla de la Figura 2.

En ambos se detecta una mínima presencia de hierro $(0.73 \%$ y $0.71 \%)$, probablemente debida a impurezas del metal. Hemos descartado la presencia de oxígeno y carbono, que atribuimos al proceso de oxidación y preparación de la muestra respectivamente. El clavo 1 (fig. 1) está completo, mide $344 \mathrm{~mm}$ de largo, es de sección cuadrada, se conserva casi recto y presenta pocas marcas de uso y desgaste. El clavo 2 (fig. 1) es un fragmento del extremo inferior, de $57 \mathrm{~mm}$ de longitud, aparentemente de sección cuadrada aunque con un alto grado de desgaste, cuya rotura pudo producirse durante el naufragio, como describen Galili et al. (2010). Otros clavos y fragmentos similares se han documentado en diferentes sectores del pecio, como los que se observan en la Figura 3.

El análisis del tercer artefacto (fig. 4) dio como resultado una aleación ternaria. Ante la heterogeneidad del material, siguiendo a Martínez-Zapata et al. (2015), se realizó una segunda medida en otra zona diferente de la muestra. Las dos zonas (A y B) dieron como resultado las composiciones que se muestran en la tabla de la Figura 5.

Los datos indican que se trata de una aleación de base cobre, en un porcentaje medio del $68,49 \%$, con estaño en una media del $2,61 \%$, y plomo en un porcentaje medio del $14,745 \%$.

Por lo que sabemos, la información en este tipo de artefactos no suele indicar la composición del bronce, por ejemplo Nieto y Foerster (1980), Foerster (1984), Beltrame y Gaddi (2005), Carre (2007), Galili et al. (2010), salvo en el caso del pecio de Ben-Afeli, excavado a principios de los años ochenta del pasado siglo, cuyos resultados indicaron un contenido de plomo del 18,61\% (Ramos et al. 1984).

\section{DISCUSIÓN}

La composición de cobre de los dos clavos evidencia lo oportuno de realizar el análisis de todos los metales para disipar dudas aportando datos que contribuyan al avance en la investigación de la arquitectura del navío. Clavos de cobre y de bronce han sido localizados en otros naufragios romanos, por ejemplo Galili et al. (2010) identificaron 911 clavos de bronce en dos pecios romanos en la costa de Carmel y 53 clavos de cobre en el pecio de Ashkelon. La determinación del metal, junto a la forma, medidas y peso, puede ayudar a clasificar estos artefactos según las tipologías propuestas, como la de Galili et al. (2010) y determinar las partes del barco en las que fueron utilizados, diferenciándolos de otros clavos fabricados con hierro o bronce.

La composición elemental del rodamiento nos invita a una reflexión sobre la eficacia de la aleación utilizada. La forma común del bronce es una combinación binaria ( $\mathrm{Sn} /$ $\mathrm{Cu}$ ) de base cobre, en la que el estaño aporta mayor resistencia y dureza, normalmente en proporciones entre un $4 \%$ 


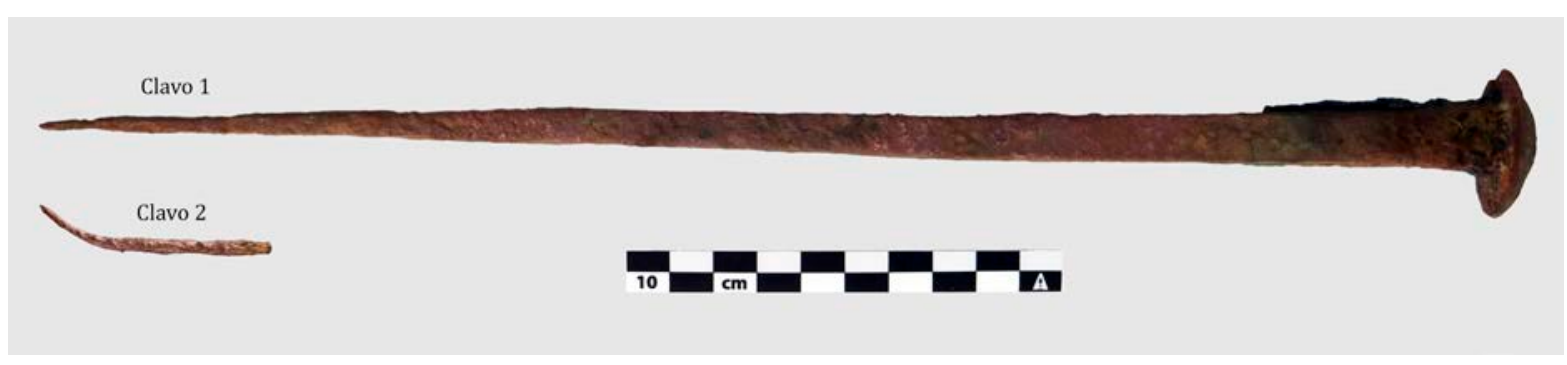

Fig. 1: Clavos 1 y 2 del pecio Bou Ferrer.

\begin{tabular}{|lcc|}
\hline El & Clavo 1 (peso: $245 \mathrm{~g})$ & Clavo 2 (peso: $3 \mathrm{~g}$ ) \\
\hline Hierro $(\mathrm{Fe})$ & $0,73 \%$ & $0,71 \%$ \\
Cobre $(\mathrm{Cu})$ & $85,43 \%$ & $88,85 \%$ \\
\hline
\end{tabular}

Fig. 2. Tabla de resultados del análisis de los clavos 1 y 2.

y un $12 \%$. La presencia de plomo, en un porcentaje relativamente alto, induce a pensar en una intencionalidad técnica para aprovechar alguna característica de esa variante de la aleación, una idea ya sugerida para los rodamientos localizados en el yacimiento de Ben-Afeli por Ramos et al (1984) que no tuvo continuidad en estudios posteriores. Actualmente, la industria del metal fabrica un tipo de bronce llamado "bronces al plomo" bajo estándares internacionales (Omamet 2017; Phemsa 2020) con porcentajes de cobre entre $78 \%$ y $82 \%$, estaño entre $9 \%$ y $11 \%$ y plomo entre $8 \%$ y $11 \%$. Como características del material resultante, Phemsa (2020) indica que esta aleación tiene grandes propiedades antifricción y una buena resistencia a la corrosión. Se utiliza para la fabricación de cojinetes en los que las condiciones de lubricación no sean perfectas, ya que el alto contenido de plomo evita el agarrotamiento. Se recomienda para cojinetes de torno, cojinetes en contacto con aguas minerales o líquidos sulfurosos. Por su parte, en Omamet (2017) se indica que las aleaciones de CuSnPb se caracterizan por su excelente cualidad antifricción, resistencia a altas presiones, corrosión, vibraciones y golpes. Se recomiendan cuando la lubricación es deficiente. Aplicaciones: bujes y cojinetes con altas cargas, prensas y palas mecánicas, bujes de biela, bombas, motores, industria automotriz y ferroviaria.

La aleación de bronce de la pieza objeto de estudio se fabricó con proporciones similares a las que se utilizan actualmente en la industria del bronce para la fabricación de piezas que resisten la fricción en ambientes corrosivos, lo que induce a pensar que el artefacto pudo fabricarse para soportar este tipo de trabajo.

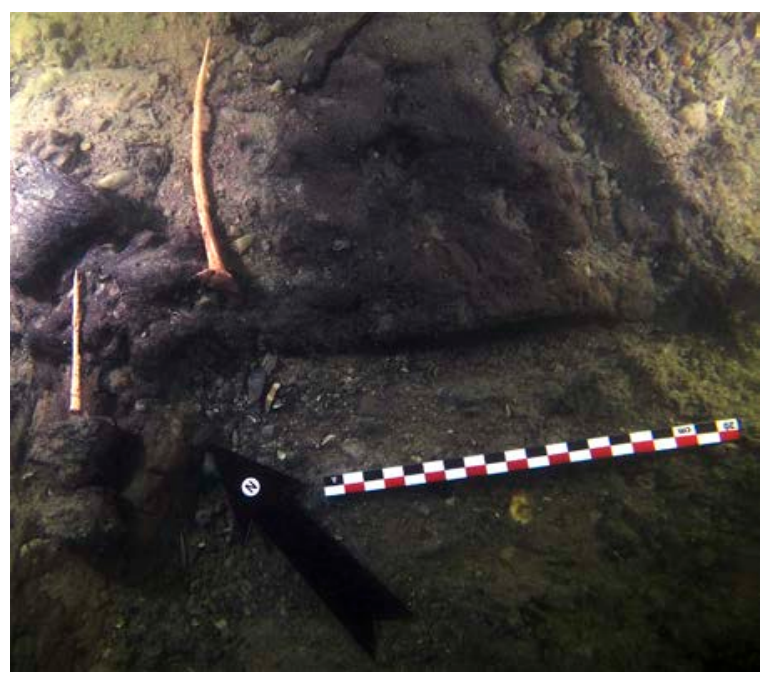

Fig. 3: Clavos en el sector O del pecio Bou Ferrer.

Carre (2007) describe diversos tipos de bomba de achique a través de los hallazgos recuperados en varios naufragios de época clásica. Siguiendo a esta autora se puede afirmar que los mecanismos de bombeo incorporaban un sistema de giro manual para elevar el agua desde la sentina hasta la cubierta. Se accionaba mediante una palanca o una manivela que movía algún tipo de polea con un rodamiento que giraba sobre un eje provisto de un casquillo, como se puede observar en la parte superior e inferior de la Figura 6, según la hipótesis de funcionamiento de Foerster (1984), que también sostienen Galili et al. (2010) con propuestas similares. La fricción constante entre el rodamiento y el casquillo o el eje, por la necesidad permanente de achicar el agua de la sentina, es lo que justificaría la necesidad de una aleación de metales con las propiedades descritas.

Autores como Nieto y Foerster (1980), Sciallano y Marlier (2008), Beltrame (2005), Carre (2007), Long (2012), también han descrito artefactos similares como mecanismos de rodamiento de las bombas de achique en 


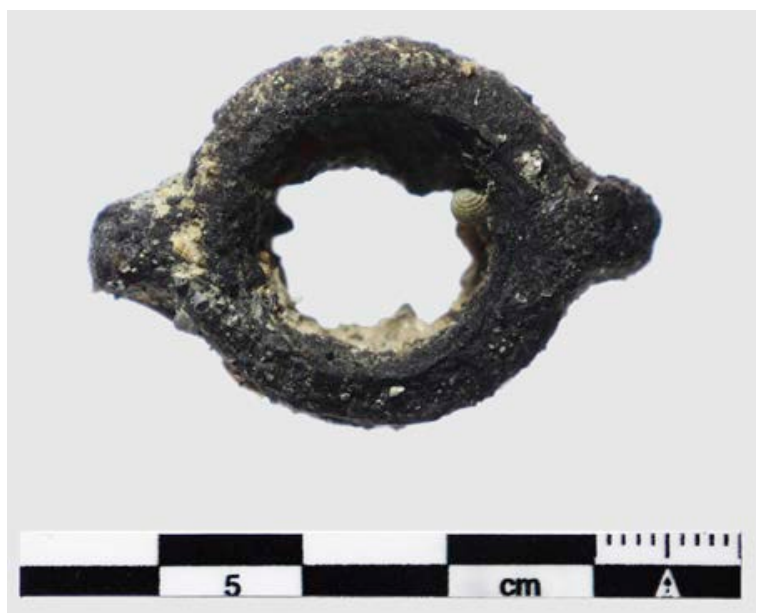

Fig. 4: Rodamiento del pecio Bou Ferrer.

\begin{tabular}{|lcccc|}
\hline El & $\begin{array}{c}\text { Rodamiento } \\
(\mathrm{A}) \%\end{array}$ & $\begin{array}{c}\text { Rodamiento } \\
(\mathrm{B}) \%\end{array}$ & Media \% & $D E$ \\
\hline Cobre $(\mathrm{Cu})$ & 68,79 & 68,19 & 68,49 & 0,424 \\
Estaño $(\mathrm{Cn})$ & 2,72 & 2,5 & 2,61 & 0,155 \\
Plomo $(\mathrm{Pb})$ & 14,37 & 15,12 & 14,745 & 0,53 \\
\hline
\end{tabular}

Fig. 5: Tabla de resultados del análisis del rodamiento.

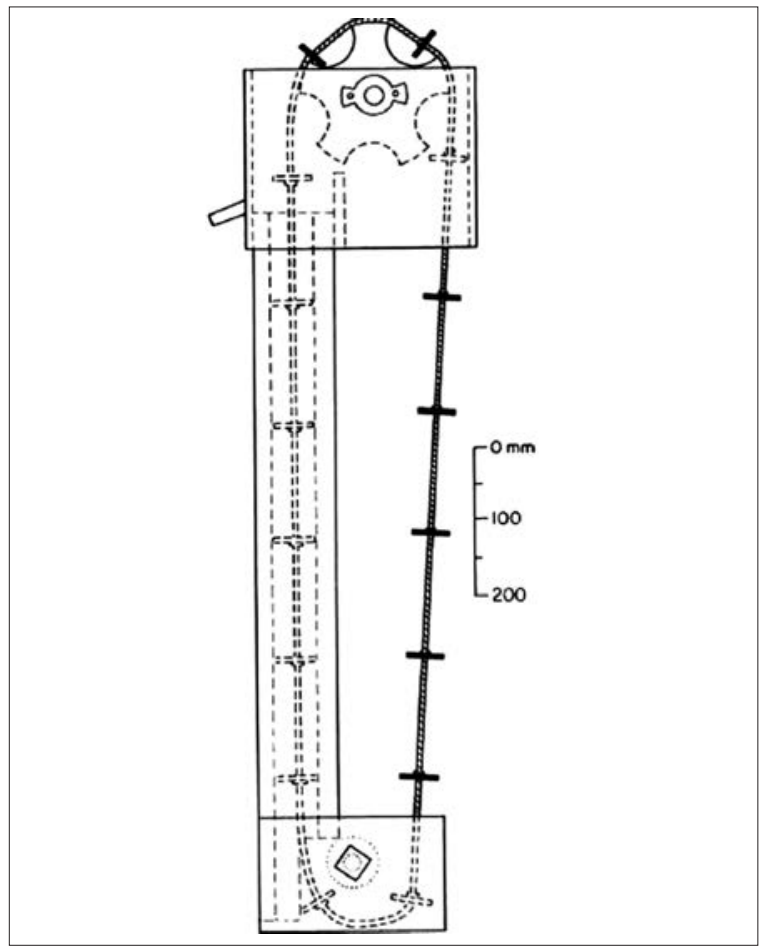

Fig. 6: Reconstrucción de la bomba de achique del naufragio de Los Ullastres (Foerster 1984: 88, fig. 4a). los pecios de Cap del Vol, Nemi, Los Ullastres, Grand Rouveau, Madrague de Giens, La Giraglia, Cap Gros o Grado. En estas investigaciones se refieren a las piezas como objetos de bronce, sin determinar el tipo de aleación o las proporciones de su composición.

Parece evidente que los rodamientos de las bombas de achique soportaban un trabajo intensivo de fricción en un ambiente corrosivo provocado por el agua de mar. Así mismo, el plomo añadido y la reducción de la proporción de estaño en una aleación ternaria de bronce lo dotan de mayor resistencia a la fricción y a la corrosión de los ambientes salinos. Por lo tanto, existe la posibilidad de que quienes fabricaron el rodamiento del pecio Bou Ferrer disponían de esos conocimientos y los aplicaron para elaborar la pieza, aunque serían necesarios más datos para confirmarlo. Como indican (Martínez-Zapata et al. 2015), la caracterización de los bronces antiguos es un reto actual y un tema de estudio aún en desarrollo; en este sentido, sería interesante continuar la investigación tomando muestras de los rodamientos de bronce depositados en museos, procedentes de otros barcos de época clásica ya excavados. El análisis de composición elemental de esas nuevas piezas de bronce podría darle la consistencia necesaria a esta línea de trabajo y establecer conclusiones definitivas.

\section{BIBLIOGRAFÍA}

BELTRAME, C.; GADDI, D. (2005): The rigging and the "hydraulic system' of the Roman wreck at Grado, Gorizia, Italy. International Journal of Nautical Archaeology 34 (1), 79-87. DOI: https://doi.org/10.1111/j.1095-9270.2005.00045.x

CARRE, M. B. (2007): Les pompes de cale et l'évacuation de l'eau de sentine sur les navires antiques, Énergie hydraulique et machines élévatrices d'eau dans l'Antiquité (Jean-Pierre Brun, Jean-Luc Fiches, dirs.), Publications du Centre Jean Bérard, <https://hal.archives-ouvertes.fr/hal-01461614> (Consulta: 19-VII-2020).

DE JUAN, C.; CIBECCHINI, F.; MOLINA, J.; MOYA, J. A.; PIQUES, G.; DOMERGE, C.; RICO, C. (2017): Estado de las investigaciones en el pecio Bou Ferrer tras las campañas financiadas por la Dirección General de Cultura (2006, 2007, 2012, 2013, 2014, 2015 y 2016): Informe inédito previo a la Campaña de Intervención en el pecio Bou Ferrer 2017, Generalitat Valenciana. Consellería d'Educació, Cultura i Sport.,València.

FERNÁNDEZ, A. (1980): Estudio de los restos arqueológicos submarinos en las costas de Castellón, CPAC 7, 135196, <http://hdl.handle.net/10234/45004> (Consulta: 20-VII-2020). 
FOERSTER, F. (1984): New views on bilge pumps from Roman wrecks, International Journal of Nautical Archaeology 13 (1), 85-93.

GALILI, E.; ROSEN, B.; SHARVIT, J. (2010a): Artifact assemblage recovered from a Roman shipwreck off the Carmel Coast, Israel, Atiqot.

GALILI, E.; SUSSMAN, V.; STIEBEL, G.; ROSEN, B. (2010b): A Hellenistic/Early Roman Shipwreck Assemblage off Ashkelon, Israel, International Journal of Nautical Archaeology 39 (1), 125-145.

DOI: https://doi.org/10.1111/j.1095-9270.2009.00249.x

LONG, L. (2012): L'épave des Riches Dunes à Marseillan: autopsie d'un contexte archéologique sous-marin encore mystérieux, Bronzes grecs et romains, recherches récentes. Hommage à Claude Rolley (M. Denoyelle, S. DescampsLequime, B. Mille, S. Verger, dirs.), INHA. DOI: https://doi.org/10.4000/books.inha.3912.

MARTÍNEZ-ZAPATA, O.; DONATE-CARRETERO, I.; ZUCCHIATTI, A.; CLIMENT-FONT, A.; BARRIO, J. (2015): Análisis no destructivo aplicado a patrimonio cultural metálico de bronce antiguo mediante la técnica PIXE, Metal España 2015. Actas del II Congreso de Conservación y Restauración del Patrimonio Metálico, Real Casa de Moneda,
Segovia, <https://digital.csic.es/handle/10261/132099> (Consulta 30-VII-2020).

MElgareJo, J. C.; PROENZA, J. A.; GALÍ, S.; LlOVET, X. (2010): Técnicas de caracterización mineral y su aplicación en exploración y explotación minera, Boletín de la Sociedad Geológica Mexicana 62 (1), 1-23, <https://www. redalyc.org/articulo.oa?id=943/94315961002> $\quad$ (Consulta 22-VII-2020).

NIETO, X.; FOERSTER, F. (1980): El pecio romano del Cap del Vol (Campañas de 1978 y 1979), Cypsela III, 163-177.

OMAMET SPA (2017): Bronces al plomo SAE64, SAE67, Chile, $<$ http://www.omamet.cl/paginas/certificacion.html> (Consulta 20-VII-2020).

PHEMSA S.L. (1983-2020). Bronces al plomo. N6 (C332/80/10/10), Madrid, <http://www.phemsa.es/?cat=8> (Consulta 20VII-2020).

RAMOS, J. E.; FERNÁNDEZ, A.; WAGNER, J. (1984): El yacimiento arqueológico submarino de Ben-Afelí. Estudio de los materiales (Almazora, Castellón), CPAC 10, 121-158.

SCIALLANO, M.; MARLIER, S. (2008): L'épave à dolia de l'île de la Giraglia (Haute-Course), Archeonáutica 15, 115-154. DOI: https://doi.org/10.3406/nauti.2008.919 